Math. Model. Nat. Phenom.

Vol. 4, No. 5, 2009, pp. 128-143

DOI: $10.1051 / \mathrm{mmnp} / 20094509$

\title{
Robust Feedback Control Design for a Nonlinear Wastewater Treatment Model
}

\author{
M. Serhani ${ }^{1, *}$, N. Raissi ${ }^{2}$ and P. Cartigny ${ }^{3}$ \\ ${ }^{1}$ FSJES, University My Ismail, B.P. 3102, Toulal, Meknes, Morocco \\ 2 EIMA, FS, University Ibn Tofail, B.P. 133, Kénitra, Morocco \\ ${ }^{3}$ GREQAM, University la Méditérranée, 2 rue de la Charité, 13002 Marseille, France
}

\begin{abstract}
In this work we deal with the design of the robust feedback control of wastewater treatment system, namely the activated sludge process. This problem is formulated by a nonlinear ordinary differential system. On one hand, we develop a robust analysis when the specific growth function of the bacterium $\mu$ is not well known. On the other hand, when also the substrate concentration in the feed stream $s_{i n}$ is unknown, we provide an observer of system and propose a design of robust feedback control in term of recycle rate, in order to keep the pollutant concentration lower than an allowed maximum level $s_{d}$.
\end{abstract}

Key words: wastewater treatment, dynamical systems, stability, robustness, feedback control, activated sludge

AMS subject classification: 92D25, 37N25, 93B07, 93B51, 93B52, 93D20

\section{Introduction}

In this paper we study a model of wastewater treatment, formulated by a nonlinear ordinary differential system. The model describes an activated sludge process in a depuration station. The working principle is described thoroughly in literature, $[5,12]$. Basically, the process can be summarized as follows : the wastewater is discharged in an aerator with a flow $Q_{i n}$ and a concentration $s_{i n}$ in the feed stream. The phase of biological oxidation of the polluted water (substrate), by a blend of one bacterial population in an aerobic reaction consuming the oxygen, begins in the aera-

*Corresponding author. E-mail: mserhani@hotmail.com 
tor and achieves in the settler. In the settler thank, the solid components will settle and concentrate at the bottom whereas the sedimentation of soluble organic matter is assumed to be not significant. A part of bacteria biomass is recycled into aerator in order to stimulate the oxidation. The main three biological phenomena considered are : the reaction kinetics in the aerator linked to microbial growth, the substrate degradation and the recycle of the biomass from the settler. A schematic of the process is shown in Fig1.

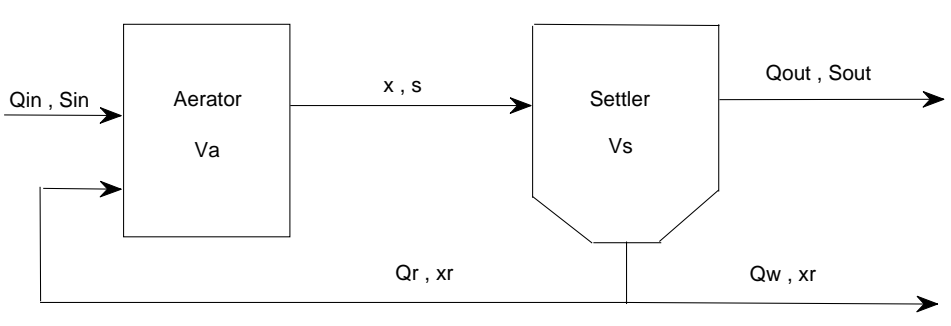

Figure 1: Schematic diagram of the activated sludge process.

Where $s, x$ and $x_{r}$ are the states variables representing respectively the substrate biomass (pollutant), the bacteria biomass and recycled bacteria biomass concentrations. $Q_{i n}, Q_{o u t}, Q_{r}, Q_{w}$ are the influent, effluent, recycle and waste flow rates, respectively. $V_{a}$ and $V_{s}$ represent the aerator and settler volumes and $s_{i n}$ corresponds to the substrate concentrations in the feed stream. Due to metabolic variations and the influence of many physical-chemical factors (PH, Temperature, oxygen, ...), it's very hard to have an accurate idea of the specific growth function of the bacterium $\mu$, (see $[10,11]$ ). On the other hand, the substrate concentration in the feed stream $s_{\text {in }}$ fluctuates and its variation depends on time and activities of the zone feeding the depuration station. Obviously, this situation incloses a very sensitive ecological problem, since due to fluctuations of $\mu$ and those of $s_{i n}$, the pollutant concentration may increase beyond the maximum tolerated level. Our goal in this work is, firstly, to carry out a robust analysis of the model showing that, even if we consider that $s_{i n}$ is constant and assume only that $\mu$ is not well known, (with given upper bound), the system admit a domain of stability instead an unique global stable equilibrium point obtained in [16] when $\mu$ is perfectly known. Secondly, when both $\mu$ and $s_{i n}$ are not known, we propose a design of robust feedback control in term of recycle rate, in order to keep the system lower than a maximum allowed level $s_{d}$. But, since $\mu$ and $s_{i n}$ are not well known, concentrations of biomasses $x, s$ and $x_{r}$ are unmeasured and hence we can't use theme to build the feedback control. Our approach consist to provide an upper observer of the system and through theory of monotone dynamical systems, we prove global partial stabilization of the upper observer of substrate concentration $s$ about $s_{d}$ and by the way keeping $s \leq s_{d}$. Many works are interested by similar problems where issues of : optimal control [12, 13], observers [1, 2, 5, 9, 10, 12, 15], simulation [6, 14], have been studied. Robust control has been also treated, $[4,5,7,8]$, but without recycle biomass. Our approach is different : firstly, our system contain a recycle phenomena. Furthermore, we use the recycle rate as control instead of dilution rate $D$, often used as control, since this last variable is practical very hard to manipulate, and varying $D$ implies variation of some other parameters as we will show 
below. On the other hand, our control do not require the knowledge of online measurement of substrate concentration $s$.

\section{Mathematical model}

The basic model is developed in $[3,5,10,12]$. The mass balance of the various constituents gives the following set of equations describing the evolution of substrate $s$, bacteria boimass $x$ and recycle bacteria biomass $x_{r}$ :

$$
(S) \quad\left\{\begin{array}{l}
\dot{s}=-\frac{\mu(s) x}{Y}-(1+r) D s+D s_{i n} ; \quad s(0)=s_{0} \\
\dot{x}=\mu(s) x-(1+r) D x+r D x_{r} ; \quad x(0)=x_{0} \\
\dot{x}_{r}=\nu(1+r) D x-\nu(w+r) D x_{r} ; \quad x_{r}(0)=x_{r 0}
\end{array}\right.
$$

with

$$
\begin{gathered}
D:=\frac{Q_{i n}}{V_{a}} \text { representing the dilution rate, } \\
r:=\frac{Q_{r}}{Q_{i n}} \text { representing the recycle rate, } \\
w:=\frac{Q_{w}}{Q_{i n}} ; \nu:=\frac{V_{a}}{V_{s}},
\end{gathered}
$$

and where $Y$ refer to the yield coefficient of the growth of biomass on substrate. $\mu($.$) is the specific$ growth function of the bacterium.

When all parameters are fully known and when $s_{i n}$ is constant and $\mu$ is monotone increasing (Monod type), we proved in Serhani et al. [16] that there exist an unique globally asymptotically stable equilibrium point. But generally, information about $\mu$ is not perfectly available, so, our goal in the next section is to investigate the situation where an upper bound of $\mu$ is handled.

Remark 1. As signaled in the introduction, the common tool through which a similar systems (bioreactors and chemostat systems) are controlled in literature is the dilution rate D, see [4, 5, 7, 15]. But in our case, manipulating $D$ implies the variation of $Q_{i n}$ since $V_{a}$ is constant. This fact leads to varying $r$ and $w$ since they depend also on $Q_{i n}$. This justify our choice, in this work (section 5), of recycle rate $r$ as control.

\section{Robustness}

The object of this section is to establish the existence of a global attractor interior domain whenever full information about growth function $\mu$ is not available. We will carry out our study in the framework of the following assumptions : 


\subsection{Assumptions}

We assume that :

$A_{1}: D, s_{i n}, r, w, \nu$ and $Y$ are positive constants.

$A_{2}:$ There exist $\bar{\mu}: R^{+} \rightarrow R^{+}$, monotone increasing and satisfying

$$
0 \leq \bar{\mu}(.) \leq \bar{m},
$$

where $\bar{m}$ is a given constant representing the above bound of $\bar{\mu}$.

$$
\begin{gathered}
\bar{\mu}(s)=0 \quad \text { if } \quad s=0 \\
\mu(s) \leq \bar{\mu}(s), \quad \forall s \geq 0 .
\end{gathered}
$$

The classic example of $\bar{\mu}$, is the so-called Monod growth function, given by the following formulation :

$$
\bar{\mu}(s):=\frac{\bar{m} s}{b+s},
$$

where $b$ is a constant representing the value at which $\bar{\mu}$ takes the half of $\bar{m}$.

Theorem 2. Assume that there exist $\bar{s}$ such that $\bar{\mu}(\bar{s})=(1+r) D \frac{w}{w+r}$ and that $\bar{s}<\frac{s_{\text {in }}}{1+r}$, then there exist $\bar{x}>0$ and $\bar{x}_{r}>0$ such that, each trajectory of system $(S)$ starting in $R_{+}^{* 3}$ converges toward the domain $D:=\left\{\left(s, x, x_{r}\right) \in R_{+}{ }^{3} /(0,0,0) \leq\left(s, x, x_{r}\right) \leq\left(\bar{s}+Y \bar{x}, \bar{x}, \bar{x}_{r}\right)\right\}$.

Inequalities must be interpreted coordinate by coordinate.

Proof. Consider the variable change $z:=x+Y s$, the system $(S)$ can be reformulated to the system $\left(S_{c}\right)$ given by

$$
\left(S_{c}\right) \quad\left\{\begin{array}{lc}
\dot{z}=-(1+r) D z+D Y s_{i n}+r D x_{r} ; & z(0)=z_{0} \\
\dot{x}=\mu\left(\frac{z-x}{Y}\right) x-(1+r) D x+r D x_{r} ; & x(0)=x_{0} \\
\dot{x}_{r}=\nu(1+r) D x-\nu(w+r) D x_{r} ; & x_{r}(0)=x_{r 0}
\end{array}\right.
$$

and consider the following systems :

$$
\begin{aligned}
& (\bar{S}) \quad\left\{\begin{array}{lc}
\dot{z}=-(1+r) D z+D Y s_{i n}+r D x_{r} ; & z(0)=z_{0} \\
\dot{x}=\bar{\mu}\left(\frac{z-x}{Y}\right) x-(1+r) D x+r D x_{r} ; & x(0)=x_{0} \\
\dot{x}_{r}=\nu(1+r) D x-\nu(w+r) D x_{r} ; & x_{r}(0)=x_{r 0},
\end{array}\right. \\
& (\underline{S}) \quad\left\{\begin{array}{l}
\dot{z}=-(1+r) D z ; \quad z(0)=z_{0} \\
\dot{x}=-(1+r) D x ; \quad x(0)=x_{0} \\
\dot{x}_{r}=-\nu(w+r) D x_{r} ; \quad x_{r}(0)=x_{r 0} .
\end{array}\right.
\end{aligned}
$$


It's clear that $(\underline{S}) \leq\left(S_{c}\right) \leq(\bar{S})$ in the sense that the vector field of $\left(S_{c}\right)$ is bounded above by the vector field of $(\bar{S})$ and below by one of the system $(\underline{S})$. On the other hand, the jacobian matrices of the systems $(\bar{S})$ and $(\underline{S})$ are respectively given by

$$
J(\bar{S})=\left(\begin{array}{ccc}
-(1+r) D & 0 & r D \\
\bar{\mu}^{\prime}\left(\frac{z-x}{Y}\right) \frac{x}{Y} & \bar{\mu}\left(\frac{z-x}{Y}\right)-\bar{\mu}^{\prime}\left(\frac{z-x}{Y}\right) \frac{x}{Y}-(1+r) D & r D \\
0 & \nu(1+r) D & -\nu(w+r) D
\end{array}\right)
$$

and

$$
J(\underline{S})=\left(\begin{array}{ccc}
-(1+r) D & 0 & 0 \\
0 & -(1+r) D & 0 \\
0 & 0 & -\nu(w+r) D
\end{array}\right) .
$$

Since the out-diagonal elements of these Jacobian matrices are nonnegative then systems $(\bar{S})$ and $(\underline{S})$ are cooperative (see [17] and [18]). Therefore, by using arguments of cooperatives inequalities (see [17] and [18]), we obtain that, if

$$
u\left(t, u_{0}\right):=\left\{\left(z(t), x(t), x_{r}(t)\right) \in R_{+}^{3} /\left(z(0), x(0), x_{r}(0)\right)=u_{0}:=\left(z_{0}, x_{0}, x_{r 0}\right)\right\}
$$

is the flow of $\left(S_{c}\right)$ and similarly $\bar{u}\left(t, u_{0}\right)$ and $\underline{u}\left(t, u_{0}\right)$ are respectively the flow of $(\bar{S})$ and $(\underline{S})$ starting at the same point $u_{0}$, then

$$
\underline{u}\left(t, u_{0}\right) \leq u\left(t, u_{0}\right) \leq \bar{u}\left(t, u_{0}\right), \quad \forall t \geq 0 .
$$

On the other hand, in the system $(\bar{S})$, the function $\bar{\mu}$ is monotone increasing and

$$
\bar{\mu}(\bar{s})=(1+r) D \frac{w}{w+r},
$$

then by using analysis developed in [16] we know that there exist a non trivial, (i.e. not lying in boundaries), equilibrium point $\bar{P}=\left(\bar{z}, \bar{x}, \bar{x}_{r}\right)$ which is globally asymptotically stable for $\bar{S}$, (this means that all trajectories of system $(\bar{S})$ starting in $R_{+}^{* 3}$ converges towards $\bar{P}$ ).

Moreover, it's very easy to see that the system $(\underline{S})$ has $\underline{P}=(0,0,0)$ as a globally asymptotically stable equilibrium point. To show this, it suffices to use the Lyapunov function

$$
V:=z^{2}+x^{2}+\left(x_{r}\right)^{2}
$$

So, according to (3.1) we conclude that all trajectories of system $\left(S_{c}\right)$ starting from a point in $R_{+}^{* 3}$ converge toward the set

$$
D:=\left\{\left(z, x, x_{r}\right) \in R_{+}^{3} /(0,0,0) \leq\left(z, x, x_{r}\right) \leq\left(\bar{z}, \bar{x}, \bar{x}_{r}\right)\right\} .
$$


It remain to conclude for the system $(S)$. Let $\left(z, x, x_{r}\right)$ be a trajectory of system $\left(S_{c}\right)$. A trajectory of $(S)$ can be derived from these of $\left(S_{c}\right)$ as

$$
\left(s:=z-Y x, x, x_{r}\right) .
$$

Recall that

$$
z \leq \bar{z} \text { and } \bar{x} \geq 0
$$

so, the substrate concentration $s$ in the system $(S)$ is such that,

$$
s \leq \bar{z},
$$

in other word,

$$
s \leq \bar{s}+Y \bar{x}
$$

where $\bar{s}=\bar{z}-Y \bar{x}$.

Furthermore, we have that

$$
x \leq \bar{x} \text { and } x_{r} \leq \bar{x}_{r}
$$

On the other hand, the trajectory $\left(s, x, x_{r}\right)$ of system $(S)$ is always nonnegative. Indeed, on the axis

$$
\left\{\left(s, x, x_{r}\right) \in \mathbb{R}_{+}^{3} / s=0\right\},
$$

we have

$$
\dot{s}=D s_{i n},
$$

so, $\dot{s}$ is positive and hence the the vector field is pointed inside $\mathbb{R}_{+}^{3}$. Similarly, on the axis

$$
\left\{\left(s, x, x_{r}\right) \in \mathbb{R}_{+}^{3} / x=0\right\},
$$

we have

$$
\dot{x}=r D x_{r} \geq 0,
$$

which implies that the vector field $\dot{x}$ is pointed inside $\mathbb{R}_{+}^{3}$. Finally, on the axis

$$
\left\{\left(s, x, x_{r}\right) \in \mathbb{R}_{+}^{3} / x_{r}=0\right\},
$$

we obtain

$$
\dot{x}_{r}=\nu(1+r) D x \geq 0,
$$

which also implies that the vector field $\dot{x}_{r}$ is pointed inside $\mathbb{R}_{+}{ }^{3}$. It follows that, on the boundaries of $\mathbb{R}_{+}{ }^{3}$, the vector field $\left(\dot{s}, \dot{x}, \dot{x}_{r}\right)$ is pointed inside the positive octant and hence each trajectory $\left(s, x, x_{r}\right)$ of system $(S)$ is such that

$$
\left(s(t), x(t), x_{r}(t)\right) \geq 0, \quad \forall t \geq 0 .
$$

We conclude from (3.2), (3.3) and (3.4), that all trajectories of the system $(S)$ converge toward the domain

$$
D:=\left\{\left(s, x, x_{r}\right) \in \mathbb{R}_{+}^{3} /(0,0,0) \leq\left(s, x, x_{r}\right) \leq\left(\bar{s}+Y \bar{x}, \bar{x}, \bar{x}_{r}\right)\right\},
$$


as required.

From the last analysis it follows that even if we suppose that the recycle rate $r$ and the substrate concentration in the feed stream $s_{i n}$ are known constants and an upper bound of $\mu$ is given, the system admit a domain of stability, instead an unique global stable equilibrium point arising when $\mu$ is perfectly known, ([16]). This situation incloses some difficulties, since, firstly, $s_{i n}$ and the boundary of $\mu$ are in general not well known. Secondly the fluctuations of the growth function $\mu$ and pollutant concentration level $s$ and hence the $s$-boundary of domain $D$ may exceed the maximum level tolerated by environment. Our goal in next sections is to control the recycle rate $r$ in order to keep $s$ lower than an allowed maximum level.

\section{Upper observer of pollutant concentration}

\subsection{Hypotheses}

Let us now formulate the new assumptions under which we will work along the rest of this work. $H_{1}$ - The coefficients $D, w, Y$ and $\nu$ are assumed to be positive constants.

$H_{2}-$ The recycled rate $r(t)$ is a known nonnegative bounded time varying function.

$H_{3}-$ We assume that $\mu$ is not known.

$H_{4}-s_{i n}(t)$ is a not well known time varying function but bounded above by :

$$
s_{\text {in }}(t) \leq s_{\text {in }}^{+}, \quad \forall t \geq 0
$$

where $s_{\text {in }}^{+}$is a given positive constant.

During the simulations in the section (6.), to be in the framework of the hypothesis $H_{3}$, we have choose a function $\mu$ with an expression, (equation (6.1)), involving oscillations, nonsmoothness and inhibition. This fact is illustrated by the figure (2). Similarly, the hypothesis $H_{4}$ is taken into account in simulation, thanks to $s_{i n}(t)$ and $s_{i n}^{+}$, given respectively by equations (6.2) and (6.3). $s_{i n}(t)$ oscillate between a known bounds for which the upper bound is $s_{i n}^{+}$as shown in figure (3).

\subsection{Observer}

To build a feedback control in term of recycle rate $r(t)$, it requiring to have a perfect knowledge of variables involved for this construction. But in practice, it is very hard to have a full information of bioprocess kinetics, so, an accurate data is not available, due to metabolic variations and the influence of many physic-chemical factors. The purpose of this section is to derive an upper observer which permit us to have an estimation of the upper bound of missing data.

Consider the following system

$$
\left(S_{u p}\right) \quad\left\{\begin{array}{l}
\dot{s}^{+}=-D(1+r(t)) s^{+}+D s_{i n}^{+} ; \quad s^{+}(0)=s_{0}^{+} \\
\dot{x}^{+}=\bar{\mu}\left(s^{+}\right) x^{+}-(1+r) D x^{+}+r D x_{r}^{+} ; x^{+}(0)=x_{0}^{+} \\
\dot{x}_{r}^{+}=\nu(1+r) D x^{+}-\nu(w+r) D x_{r}^{+} ; x_{r}^{+}(0)=x_{r 0}^{+},
\end{array}\right.
$$


we obtain an upper observer of the system $(S)$.

Theorem 3. For given nonnegative vectors $\left(s_{0}^{+}, x_{0}^{+}, x_{r 0}^{+}\right)$and $\left(s_{0}, x_{0}, x_{r 0}\right)$ such that $\left(s_{0}, x_{0}, x_{r 0}\right) \leq$ $\left(s_{0}^{+}, x_{0}^{+}, x_{r 0}^{+}\right)$, we have

$$
s(t) \leq s^{+}(t), x(t) \leq x^{+}(t), x_{r}(t) \leq x_{r}^{+}(t), \forall t \geq 0 .
$$

Where $\left(s(t), x(t), x_{r}(t)\right)$ and $\left(s^{+}(t), x^{+}(t), x_{r}^{+}(t)\right)$ are the vector fields of systems $(S)$ and $\left(S_{u p}\right)$ respectively with initial conditions $\left(s_{0}, x_{0}, x_{r 0}\right)$ and $\left(s_{0}^{+}, x_{0}^{+}, x_{r 0}^{+}\right)$respectively.

Proof. Let $p(t)=\left(p_{1}(t), p_{2}(t), p_{3}(t)\right) \in R_{3}^{+}, \forall t \geq 0$ and $q(t)=\left(q_{1}(t), q_{2}(t), q_{3}(t)\right) \in$ $R_{3}^{+}, \forall t \geq 0$. By choosing

$$
f(t, p(t)):=\left\{\begin{array}{l}
-\frac{\mu\left(p_{1}(t)\right)}{Y} p_{2}(t)-(1+r) D p_{1}(t)+D s_{i n} \\
\mu\left(p_{1}(t)\right) p_{2}(t)-(1+r) D p_{2}(t)+r D p_{3}(t) \\
\nu(1+r) D p_{2}(t)-\nu(w+r) D p_{3}(t)
\end{array}\right.
$$

and

$$
g(t, q(t)):=\left\{\begin{array}{l}
-D(1+r) q_{1}(t)+D s_{i n}^{+} \\
\bar{\mu}\left(q_{1}(t)\right) q_{2}(t)-(1+r) D q_{2}(t)+r D q_{3}(t) \\
\nu(1+r) D q_{2}(t)-\nu(w+r) D q_{3}(t) .
\end{array}\right.
$$

The systems $(S)$ and $\left(S_{u p}\right)$ can be rewritten as

$$
\dot{p}(t)=f(t, p(t)) ; p(0)=p_{0}
$$

and

$$
\dot{q}(t)=g(t, q(t)) ; \quad q(0)=q_{0}
$$

Where

$$
p(t)=\left(s(t), x(t), x_{r}(t)\right), q(t)=\left(s^{+}(t), x^{+}(t), x_{r}^{+}(t)\right), \forall t \geq 0
$$

and

$$
p_{0}=\left(s_{0}, x_{0}, x_{r 0}\right), q_{0}=\left(s_{0}^{+}, x_{0}^{+}, x_{r 0}^{+}\right) .
$$

It's clear, according to hypothesis $H_{4}$, that for all $t, p(t) \geq 0$,

$$
f(t, p(t)) \leq g(t, p(t))
$$

Hence, we obtain, according to cooperative property of system 4.3 , that, for a vectors fields $p$ and $q$ of systems 4.2 and 4.3 respectively, if the initial conditions satisfying $p_{0} \leq q_{0}$ then the following inequality holds

$$
p(t) \leq q(t), \forall t \geq 0
$$


We conclude according to this last inequality (4.4) and the fact

$$
\left(s_{0}, x_{0}, x_{r 0}\right) \leq\left(s_{0}^{+}, x_{0}^{+}, x_{r 0}^{+}\right)
$$

that

$$
s(t) \leq s^{+}(t), x(t) \leq x^{+}(t), x_{r}(t) \leq x_{r}^{+}(t), \quad \forall t \geq 0
$$

as required.

\section{Control action}

The main of this section is to resolve the following question $(Q)$ :

Given a initial condition $\left(s_{0}, x_{0}, x_{r 0}\right) \in \mathbb{R}_{+}^{3 *}$, find a feedback control, in term of recycle rate $r(t)$, to ensure that, for any unknown $\mu$ and $s_{\text {in }}$ fulfilling $H_{3}$ and $H_{4}$, the pollutant concentration $s$ of system $(S)$ be lower than a maximum fixed level $s_{d}$.

Let the feedback law $r$ given by the following expression

$$
r\left(s^{+}, s_{i n}^{+}\right)=\left\{\begin{array}{l}
\frac{1}{s_{d}}\left(s_{i n}^{+}-s_{d}\right), \text { if } s^{+}<s_{d} \\
\frac{1}{D s^{+}}\left[\left(s_{i n}^{+}-s^{+}\right) D+\lambda\left(s^{+}-s_{d}\right)\right], \text { if } s^{+} \geq s_{d},
\end{array}\right.
$$

where $s^{+}$is the upper observer of $s$ given by (4.2.) and $\lambda \in \mathbb{R}$.

To simplify notation we will use the notation $r(t)$. Let us first prove that $r(t)$ is nonnegative and bounded.

Proposition 4. If $s_{d}<s_{i n}^{+}(t)$ and $\lambda>D$ then the control $r(t)$ given by (5.1), is nonnegative and bounded.

Proof. If $s^{+} \leq s_{d}$ then $r(t)=\frac{1}{s_{d}}\left(s_{i n}^{+}-s_{d}\right)$, but since $s_{d} \leq s_{i n}^{+}$, we obtain that $r(t) \geq 0$.

Now if $s^{+}>s_{d}$ then $r(t)=\frac{1}{D s^{+}}\left[\left(s_{i n}^{+}-s^{+}\right) D+\lambda\left(s^{+}-s_{d}\right)\right]$.

We have that $r(t) \geq 0$ if

$$
\left(s_{i n}^{+}-s^{+}\right) D+\lambda\left(s^{+}-s_{d}\right) \geq 0,
$$

which is equivalent to

$$
\lambda \geq D \frac{s^{+}(t)-s_{i n}^{+}(t)}{s^{+}(t)-s_{d}}, \forall t \geq 0 .
$$

It suffices to choose

$$
\lambda \geq \max _{t \geq 0}\left\{D \frac{s^{+}(t)-s_{i n}^{+}(t)}{s^{+}(t)-s_{d}}\right\}=D .
$$


Therefore, to have $r(t) \geq 0$, it suffice to choose

$$
\lambda \geq D
$$

which is the case by assumption.

The fact that $r(t)$ is bounded, is obtained by studying variation of $r(t)$ with respect $s^{+}$. By simple arguments of real functions variation study, we obtain that, if $\lambda \geq \frac{s_{i n}^{+}}{s_{d}}$, then

$$
r(t) \in\left[\lambda-D, \frac{s_{i n}^{+}-s_{d}}{s_{d}}\right]
$$

and if $\lambda \leq \frac{s_{i n}^{+}}{s_{d}}$, then

$$
r(t) \in\left[\frac{s_{i n}^{+}-s_{d}}{s_{d}}, \lambda-D\right]
$$

as required.

The nonnegativity of the feedback control indicates that our choice is correct while the fact that it is bounded is very important since, in practice the quantities recycled are finished. Those properties are illustrated through simulations, in the next section, for two choices of the parameter $s_{d}\left(s_{d}=\right.$ 0.5 and $\left.s_{d}=0.05\right)$, see figure (8).

Now we give our mean result.

Theorem 5. With feedback control $r(t)$ given by equation (5.1), there exist $T \geq 0$, such that, for all $t \geq T, s(t) \leq s_{d}$.

Where $s$ is the biomass concentration of substrate.

Proof. The idea is to stabilize the upper observer $s^{+}$about $s_{d}$, and since $s(t) \leq s^{+}(t), \forall t \geq 0$, we obtain that $s(t) \leq s_{d}, \forall t \geq 0$. Prove now that with the choice of the recycle rate $r(t)$ as given by equation (5.1), $s^{+}$is globally asymptotically stable about $s_{d}$.

Consider the Lyapunov function

$$
V\left(s^{+}\right):=\left(s^{+}-s_{d}\right)^{2} .
$$

$V$ is nonnegative and $V\left(s_{d}\right)=0$.

Prove now that $\frac{d V}{d t}\left(s^{+}\right)<0$, for all $s^{+} \neq s_{d}$.

$$
\begin{aligned}
\frac{d V}{d t}\left(s^{+}\right) & =2\left(s^{+}-s_{d}\right) \frac{d}{d t} s^{+} \\
& =2\left(s^{+}-s_{d}\right)\left(-D(1+r(t)) s^{+}+D s_{i n}^{+}(t)\right) .
\end{aligned}
$$

If $s^{+}<s_{d}$ then

$$
r(t)=\frac{1}{s_{d}}\left(s_{i n}^{+}-s_{d}\right) .
$$


It follows that,

$$
\begin{aligned}
\frac{d V}{d t} & =2\left(s^{+}-s_{d}\right)\left(-D \frac{s_{i n}^{+}}{s_{d}} s^{+}+D s_{i n}^{+}\right) \\
& =2\left(s^{+}-s_{d}\right) D s_{i n}^{+}\left(-\frac{s^{+}}{s_{d}}+1\right)
\end{aligned}
$$

But, $\frac{s^{+}}{s_{d}}<1$, then, since $\left(s^{+}-s_{d}\right)<0$ and $-\frac{s^{+}}{s_{d}}+1>0$, we obtain that

$$
\frac{d V}{d t}\left(s^{+}\right)<0, \forall s^{+} \neq s_{d}
$$

as required.

If now $s^{+} \geq s_{d}$ then

So,

$$
r(t)=\frac{1}{D s^{+}}\left[\left(s_{i n}^{+}-s^{+}\right) D+\lambda\left(s^{+}-s_{d}\right)\right]
$$

$$
\frac{d V}{d t}\left(s^{+}\right)=-2 \lambda\left(s^{+}-s_{d}\right)^{2}<0, \quad \forall s^{+} \neq s_{d}
$$

as required.

The simulations in the next section (6.) give an illustration of the theoretical investigations of the previous theorem. Firstly, the fact that the upper observer $s^{+}$can be stabilized about $s_{d}$, through the feedback control (5.1), (as established in the proof of the previous theorem), is illustrated by the figure (4) in which we show that $s^{+}$is stabilizated about $s_{d}$ for two values : $s_{d}=0.5$ and $s_{d}=0.05$. On the other hand, the figure (5) illustrate the result of the previous theorem and shows that the substrat $s$ is keeping below the allowed level $s_{d}$, (for $s_{d}=0.5$ and $s_{d}=0.05$ ). Finally, figures (6) and (7) show the evolution of the bacteria biomass $x$ and recycle biomass $x_{r}$ in time when we use the feedback control $r$ given by equation (5.1), for respectively $s_{d}=0.05$ and $s_{d}=0.5$.

\section{Numerical simulations}

Simulation results are obtained for the following specific growth function

$$
\mu(s):=\frac{400 s}{75+s+\frac{s^{2}}{100}}|\sin (s)|+\frac{s}{20} \sin (2 s),
$$

In this choice, the sinus function is used to produce periodicity and absolute value is used to produce non smoothness and finally the quadratic term to produce inhibition.

The concentration in feed stream is given by

$$
s_{i n}(t):=5+\sin (2 t)+\sin (20 t) .
$$


An upper bound, $s_{i n}^{+}$, of $s_{i n}(t)$ can hence be equal to

$$
s_{i n}^{+}=7
$$

The model parameters and initial conditions are given as follows :

$D=0.3 ; Y=0.8 ; \nu=2 ; w=0.5 ; x_{0}=1225 ; x_{r 0}=2333 ; s_{0}=20 ; \lambda=2 ; s_{0}^{+}=30$.

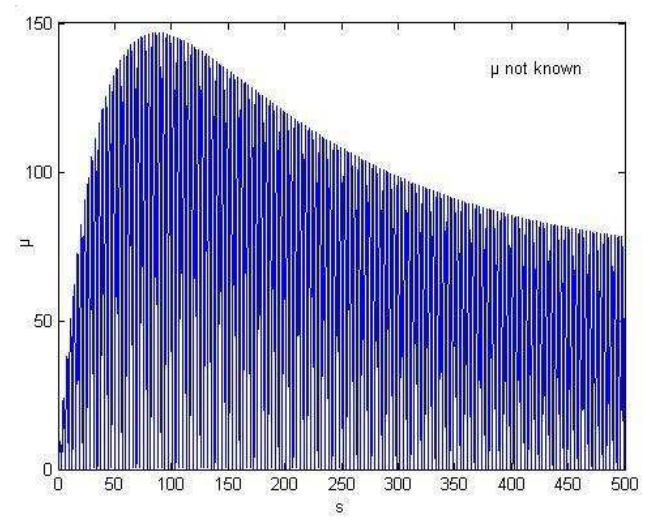

Figure 2: Variation of the unknown specific growth function $\mu($.$) .$

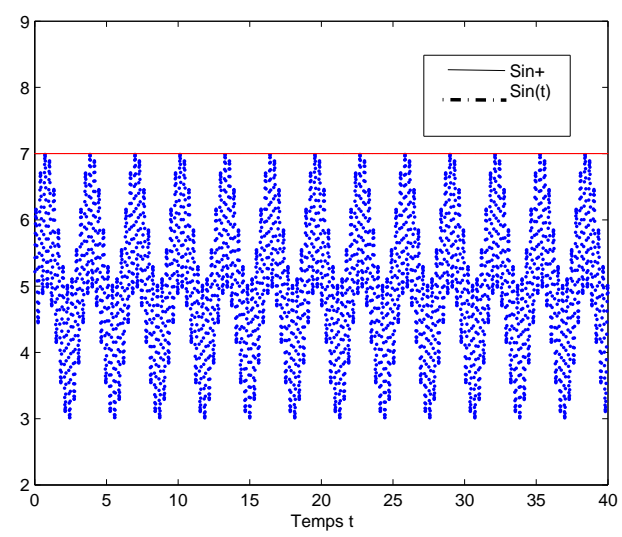

Figure 3: Variation of the uncertain concentration in feed stream $s_{i n}$. 


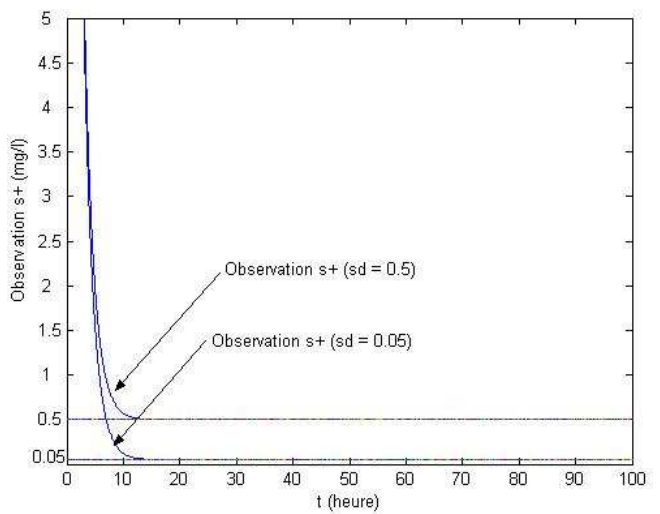

Figure 4: Graph of upper observer $s^{+}$.

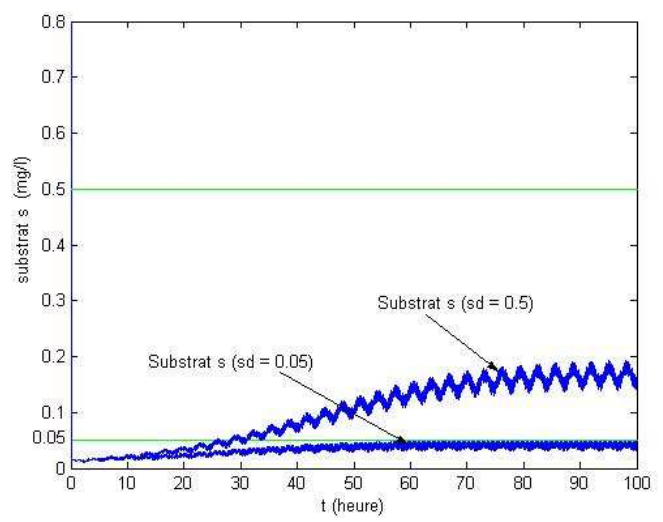

Figure 5: Graph of pollutant concentration $s$.

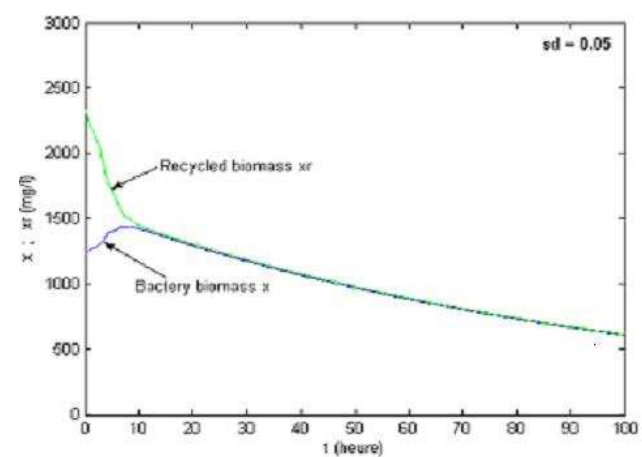

Figure 6: Bacterium biomass and recycle biomass for $(s d=0.05)$. 


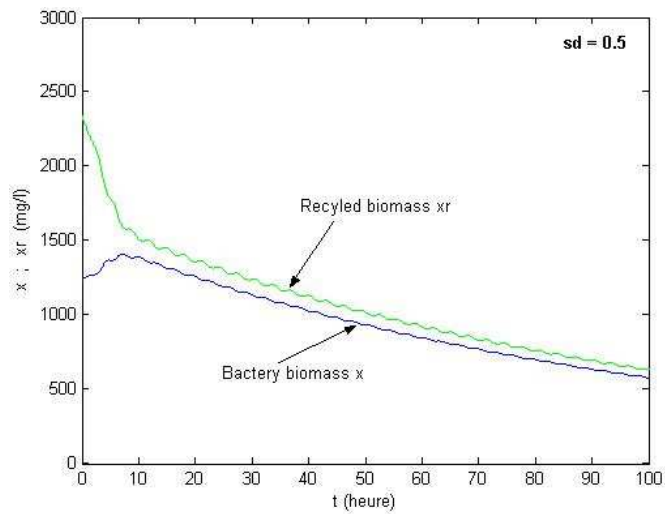

Figure 7: Bacterium biomass and recycled biomass for $(s d=0.5)$.

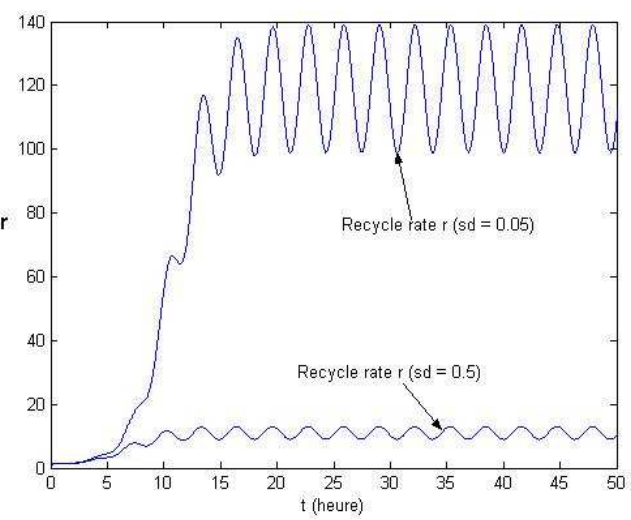

Figure 8: Variation of control $r($.$) .$ 


\section{Conclusion}

This work is interested by an activated sludge problem. We developed a robust analysis when the specific growth function of the bacteria $\mu$ is not well known, leading to existence of a stability domain. When also the substrate concentrations in the feed stream $s_{i n}$ is not known, we built a feedback control, in terms of recycle rate $r($.$) , depending on an observer of bacteria concentration$ $s$ (pollutant), in order to keep this last variable below than an allowed maximum level $s_{d}$.

\section{Acknowledgements}

We are grateful to the anonymous referee for his valuable comments suggestions and remarks.

\section{References}

[1] V. Alcaraz-Gonzalez, J. Harmand, A. Rapaport, J.-P. Steyer, V. Gonzalez Alvarez, C. Pelayo Ortiz. Application of a robust interval observer to an anaerobic digestion process. Developments in Chemical Engineering Mineral Processing, 13 (2005), No. 3/4, 267-278.

[2] V. Alcaraz-Gonzalez, J. Harmand, A. Rapaport, J.-P. Steyer, V. Gonzalez Alvarez, C. Pelayo Ortiz. Robust interval-based regulation for anaerobic digestion processes. Water Science and Technology, 52 (2005), No. 1-2, 449-456.

[3] J.F. Andrews. Kinetic models of biological waste treatment process. Biotech. Bioeng. Symp., 2 (1971), 5-34.

[4] J.F. Andrews. Dynamics and control of wastewater systems. Water quality management library, vol. 6, 1998.

[5] J.F. Busb, J.F. Andrews. Dynamic modelling and control strategies for the activated sludge process. J. of Wat. Pollut. Control Fed., 47 (1975), 1055-1080.

[6] C.R. Curds. Computer simulation of microbial population dynamics in the activated sludge process. Water Research 5 (1971), 1049-1066.

[7] D. Dochain, M. Perrier. Control design for nonlinear wastewater treatment processes. Wat. Sci. Tech. 11-12 (1993), 283-293.

[8] D. Dochain, M. Perrier. Dynamical modelling, analysis, monitoring and control design for nonlinear bioprocess. T. Scheper (Ed.), Advances in Biochemical and Biotechnology, 56 (1997), Spring Verlag, Berlin, 147-197. 
[9] C. Gómez-Quintero, I. Queinnec. Robust estimation for an uncertain linear model of an activated sludge process. Proc. of the IEEE Conference on Control Applications (CCA), Glasgow (UK), (2002), 18-20 december.

[10] M.Z. Hadj-Sadok, J.L. Gouzé. Estimation of uncertain models of activated sludge processes with interval observers. J. of Process Control, 11 (2001), 299-310.

[11] B. Haegeman, C. Lobry, J. Harmand. Modeling bacteria flocculation as density-dependent growth. Aiche Journal, 53 (2007), No.2, 535-539.

[12] S. Marsili-Libelli. Optimal control of the activated sludge process. Trans. Inst. Meas. Control, 6 (1984), 146-152.

[13] A. Martínez, C. Rodríguez, M.E. Vázquez-Méndez. Theoretical and numerical analysis of an optimal control problem related to wastewater treatment. SIAM J. CONTROL OPTIM., Vol. 38 (2000), No. 5, 1534-1553.

[14] M.K. Rangla, K.J. Burnham, L.Coyle, R.I.Stephens. Simulation of activated sludge process control strategies. Simulation '98. International Conference on (Conf. Publ. No. 457), 30 Sep - 2 Oct (1998), 152-157.

[15] A. Rapaport, J. Harmand. Robust regulation of a class of partially observed nonlinear continuous bioreactors. J. of Process Control, 12 (2002), No. 2, 291-302.

[16] M. Serhani, J.L. Gouzé, N. Raïssi. Dynamical study and robustness for a nonlinear wastewater treatment model. Proceeding book "Systems Theory : Modeling, Analysis \& Control, FES2009”, Eds A. EL Jaï, L. Afifi \& E. Zerrik, PUP, ISBN 978-2-35412-043-6, pp. 571-578.

[17] H.L. Smith. Monotone dynamical systems : an introduction to the theory of competitive and cooperative systems. American Matheatical society, 1995.

[18] H.L. Smith, P. Waltman. The theory of the chemostat. Cambridge University Press, Cambridge, 1995. 